\title{
Cytotoxicity of Novel Hydrazide-hydrazone Derivatives towards Tumor and Normal Cell Lines
}

\author{
Mayssoune Y. Zaki \\ National Organization for Drug Control \& Research \\ (NODCAR) P.O. 29 Cairo, Egypt.
}

\begin{abstract}
7 HE REACTION of cyanoacetyl hydrazone with ninhydrin gave 1 the hydrazide - hydrazone derivative 3 . The reactivity of the later product towards different chemical reagents was studied. The cytotoxicity of the newly synthesized products was measured towards the three cancer cell lines, namely breast adenocarcinoma (MCF-7), non-small cell lung cancer(NCI-H460) and CNS cancer (SF-268).
\end{abstract}

Keywords: Ninhydrin, Hydrazide-hydrazone, Pyridine and Pyridazone.

The hydrazone group has been known for its antimicrobial activity and a number of hydrazide-hydrazones were claimed to possess interesting antibacterial, antifungal $^{(1-3)}$, anticonvulsant ${ }^{(4-6)}$, anti-inflammatory ${ }^{(7,8)}$ antimalarial $^{(9)}$ and antitubercular activities ${ }^{(10-15)}$.

\section{Results and Discussion}

\section{Chemistry}

We herein report the synthesis of a series of biologically-active hydrazidehydrazones whose precursor, the hydrazide-hydrazone derivative $3^{(16)}$, was obtained via the reaction of cyanoacetic acid hydrazide (1) with ninhydrin (2) .

The structure of the latter product was based on analytical and spectral data. Thus, the $\mathrm{H}^{1} \mathrm{NMR}$ spectrum of the reaction product showed a singlet at $\delta 3.82$ corresponding to the active methylene group, a singlet at $\delta 8.80$ for the $\mathrm{NH}$ group, and a multiplet at $\delta 7.30-7.39$ resulting from the four $\mathrm{C}_{6} \mathrm{H}_{4}$ protons.

Further structural confirmation of compound 3 was achieved through studying its reactivity towards several chemical reagents. Thus, the reaction of compound 3 with benzaldehyde (4) gave the benzal derivative 5, whose analytical and spectral data are in agreement with the proposed structure. It is noteworthy that many arylidine derivatives were previously obtained via the reaction of aromatic aldehydes with cyanomethylene reagents ${ }^{(17-19)}$.

Compound 5 has reacted with either malononitrile (6a) or ethyl cyanoacetate (6b) to afford pyridine derivatives 8a,b, respectively (Scheme 1). Structure elucidation for the latter products was based on analytical and experimental data (see Experimental section). 
<smiles>N#CCC(=O)NN=c1c(=O)c2ccccc2c1=O</smiles><smiles>COC(=O)c1ccccc1/C=C(\C)C(=O)NNN1C(=O)c2ccccc2C1=O</smiles><smiles>[Y]CCNC(=O)NN=c1c(=O)c2ccccc2c1=O</smiles><smiles>[X]C(N)C(=O)C(=O)NNC1C(=O)c2ccccc2C1=O</smiles><smiles>[X]C1=C(N)C(=Cc2ccccc2)C(=O)N(N2C(=N)C(=O)c3ccccc3C2=O)C1=O</smiles>

sa, $\mathrm{X}=\mathrm{CN}$
$\mathrm{b}, \mathrm{X}=\mathrm{COOEt}$

Scheme 1 .

On the other hand, the reaction of compound 5 with either hydrazine hydrate (9a) or phenyl hydrazine (9b) gave the pyrazole derivatives $10 \mathrm{a}, \mathrm{b}$, respectively. 
The reaction of compound 3 with benzenediazonium chloride (11) in ethanol/sodium acetate at $0-5{ }^{\circ} \mathrm{C}$ gave the hydrazone derivative 12 (Scheme 2). Compound 12 reacted with either malononitrile (6a) or ethyl cyanoacetate (6b) and afforded products with molecular formulae $\mathrm{C}_{21} \mathrm{H}_{13} \mathrm{~N}_{7} \mathrm{O}_{3}$ and $\mathrm{C}_{23} \mathrm{H}_{18} \mathrm{~N}_{6} \mathrm{O}_{5}$, respectively. Two possible isomeric structures were proposed for the mentioned structures; either $14 \mathrm{a}, \mathrm{b}$ or $15 \mathrm{a}, \mathrm{b}$ (Scheme 3 ). Structures $14 \mathrm{a}, \mathrm{b}$ were considered for the reaction products based on the $\mathrm{H}^{1} \mathrm{NMR}$ data which asserted the presence of the -CO-NH- group of the hydrazone moiety through the chemical shift at $\delta$ 8.72-8.83, together with the absence of the shift characteristic of the phenyl hydrazo NH group which would have been eminent in the spectra of $15 \mathrm{a}, \mathrm{b}$.
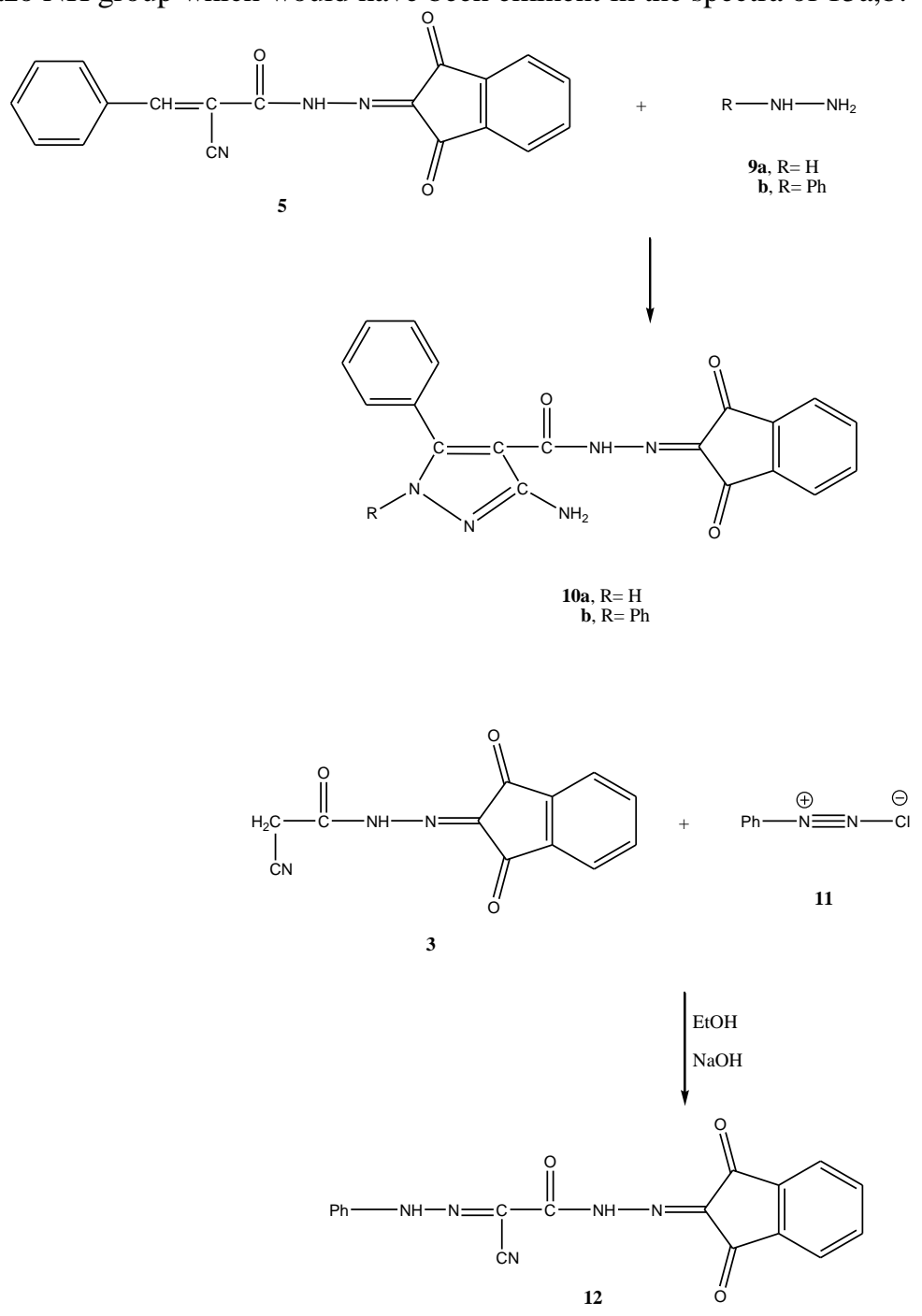

Scheme 2. 
<smiles>[X]CCNC(=O)/C(C#N)=N/NC(=O)/C(N)=N/Nc1ccccc1</smiles>

12

6a, $\mathrm{X}=\mathrm{CN}$<smiles>[3H][13CH3]</smiles><smiles>[X]C(N)=C(N)/C(=N\Nc1ccccc1)C(=O)NN=c1c(=O)c2ccccc2c1=O</smiles><smiles>[X]c1c(N)c(C(=O)NN=c2c(=O)c3ccccc3c2=O)nn(-c2ccccc2)c1=N</smiles>

Scheme 3.<smiles>[X]C1=C(N)/C(=N\Nc2ccccc2)C(=O)N(N=c2c(=O)c3ccccc3c2=O)C1=N</smiles>

$15 a, \mathrm{X}=\mathrm{CN}$

\section{Biological evaluation}

TABLE 1. Effect of the obtained compounds on the growth of three human tumor cell lines.

\begin{tabular}{llll}
\hline Compound & \multicolumn{2}{c}{$\mathbf{I C}_{\mathbf{5 0}}\left(\boldsymbol{\mu m o l ~ \mathbf { L } ^ { - \mathbf { 1 } } )}\right.$} \\
\cline { 2 - 3 } & $\mathbf{M C F - 7}$ & $\mathbf{N C I - H 4 6 0}$ & SF-268 \\
\hline $\mathbf{3}$ & $30.4 \pm 2.8$ & $20.1 \pm 4.6$ & $36.3 \pm 4.5$ \\
$\mathbf{5}$ & $0.01 \pm 0.008$ & $0.01 \pm 0.006$ & $0.08 \pm 0.08$ \\
$\mathbf{8 a}$ & $77.8 \pm 10.0$ & $64.2 \pm 8.4$ & $70.2 \pm 12.6$ \\
$\mathbf{8 b}$ & $34.2 \pm 12.6$ & $33.7 \pm 6.6$ & $44.2 \pm 8.2$ \\
$\mathbf{1 0 a}$ & $32.0 \pm 2.5$ & $24.0 \pm 4.6$ & $26.5 \pm 2.8$ \\
$\mathbf{1 0 b}$ & $10.0 \pm 0.8$ & $8.3 \pm 2.8$ & $16.5 \pm 4.0$ \\
$\mathbf{1 2}$ & $44.4 \pm 6.8$ & $26.1 \pm 2.6$ & $34.3 \pm 2.5$ \\
$\mathbf{1 4 a}$ & $0.01 \pm 0.008$ & $0.01 \pm 0.006$ & $0.08 \pm 0.08$ \\
$\mathbf{1 4 b}$ & $70.8 \pm 10.0$ & $66.2 \pm 8.4$ & $74.2 \pm 12.6$ \\
Doxorubicin & $0.04 \pm 0.008$ & $0.09 \pm 0.008$ & $0.09 \pm 0.007$
\end{tabular}

Results are given in concentrations that were able to cause $50 \%$ of cell growth inhibition $\left(\mathrm{GI}_{50}\right)$ after a continuous exposure of $48 \mathrm{hr}$ and show means \pm SEM of three-independent experiments performed in duplicate.

Egypt. J. Chem. 56, No. 1 (2013) 


\section{Structure activity relationship}

Ten compounds were tested towards the three cancer cell lines namely breast adenocarcin oma (MCF-7), non-small cell lung cancer (NCI-H460) and CNS cancer (SF-268). It is obvious from Table 1 that compound 5 which is 2-cyano$\mathrm{N}^{\prime}$-(1,3-dioxo-1H-inden-2(3H)-ylidene)-3-phenylacrylohydrazide and 14a which is $\quad 4$-amino-5-cyano- $\mathrm{N}^{\prime}$-(1,3-dioxo- $1 \mathrm{H}$-inden-2(3H)-ylidene)-6-imino-1-phenyl1,6-dihydropyridazine-3-carbohydrazide showed the maximum inhibition effect towards the three cancer cell lines and such inhibition is much higher than the reference doxorubicin. Among the tested compounds, $10 \mathrm{~b}$ which is 3 -amino-N'(1,3-dioxo-1H-inden-2(3H)-ylidene)-1,5-diphenyl-1H-pyrazole-4-

carbohydrazide showed moderate activity. However, compounds 3, 8a, 8b, 10a, 12 and $14 \mathrm{~b}$ showed the least activity towards the three cancer cell lines.

\section{Experimental}

\section{Chemistry}

All melting points were determined in open capillaries and are uncorrected. IR spectra were measured using $\mathrm{KBr}$ discs on a Pye Unicam SP-1000 spectrophotometer. ${ }^{1} \mathrm{H}-\mathrm{NMR}$ spectra were measured on a Varian EM390-200 $\mathrm{MHz}$ instrument in $\mathrm{CD}_{3} \mathrm{SOCD}_{3}$ as solvent using TMS as internal standard, and chemical shifts are expressed as $\delta$ in units of parts per million (ppm). Analytical data were obtained from the Micro analytical Data Unit at Cairo University.

\section{2-Cyano- $N^{\prime}-(1,3-$ dioxo-1H-inden-2(3H)-ylidene)acetohydrazide (3)}

To a solution of cyanoacetic acid hydrazide (1) $(6.00 \mathrm{~g}, 0.061 \mathrm{~mol})$ in $80 \mathrm{ml}$ of 1,4-dioxane, ninhydrin (2) (10.79 g, $0.061 \mathrm{~mol})$ was added and the reaction mixture subjected to heat under reflux for $3 \mathrm{hr}$, after which it was poured on a mixture of ice and water and a few concentrated $\mathrm{HCl}$ drops were added to enhance precipitation. The formed precipitate was then collected by suction filtration.

Orange crystals from ethanol, yield $84.94 \%, 12.50$ g, m.p. $225-228{ }^{\circ} \mathrm{C}$. IR $(\mathrm{KBr}): \mathrm{v} / \mathrm{cm}^{-1}=3470-3321(\mathrm{NH}), 2892\left(\mathrm{CH}_{2}\right), 3059(\mathrm{CH}$ aromatic $), 2258(\mathrm{CN})$, 1693-1680 (3CO), $1660(\mathrm{C}=\mathrm{N}), 1644(\mathrm{C}=\mathrm{C}) . \mathrm{H}^{1} \mathrm{NMR}(\mathrm{DMSO}) \delta=3.82(\mathrm{~s}, 2 \mathrm{H}$, $\mathrm{CH}_{2}$ ), 7.30-7.39 (m, 4H, $\left.\mathrm{C}_{6} \mathrm{H}_{4}\right), 8.80(\mathrm{~s}, 1 \mathrm{H}, \mathrm{NH})$. Calc. for $\mathrm{C}_{12} \mathrm{H}_{7} \mathrm{~N}_{3} \mathrm{O}_{3}(241.20)$ : C, 59.75; H, 2.93; N, $17.42 \%$. Found: C, 59.88; H, 3.01; N, $17.20 \%$.

2-Cyano- $N^{\prime}-(1,3-$ dioxo-1H-inden-2(3H)-ylidene)-3-phenylacrylohydrazide (5)

Compound $3\left(2.00 \mathrm{~g}, 8.292 \times 10^{-3} \mathrm{~mol}\right)$ has been dissolved in $50 \mathrm{ml}$ of $1,4-$ dioxane and benzaldehyde $\left(0.88 \mathrm{~g}, 8.292 \times 10^{-3} \mathrm{~mol}\right)$ and $0.5 \mathrm{ml}$ of piperidine stirred in. The reactants were then heated under reflux for $2 \mathrm{hr}$ and then poured on ice and a few drops of concentrated $\mathrm{HCl}$ were added to enhance precipitation. The formed precipitate was then filtered out.

Pale yellow crystals from ethanol, yield $73.23 \%, 2.00$ g, m.p. $256-259{ }^{\circ} \mathrm{C}$. IR $(\mathrm{KBr}): \mathrm{v} / \mathrm{cm}^{-1}=3482-3340(\mathrm{NH}), 3055(\mathrm{CH}$ aromatic), $2256(\mathrm{CN}), 1690-1683$ 
(3CO), $1656(\mathrm{C}=\mathrm{N}), 1640(\mathrm{C}=\mathrm{C}) . \mathrm{H}^{1} \mathrm{NMR}(\mathrm{DMSO}) \delta=6.58(\mathrm{~s}, 1 \mathrm{H}, \mathrm{CH}), 7.26-$ $7.38\left(\mathrm{~m}, 9 \mathrm{H}, \mathrm{C}_{6} \mathrm{H}_{5}, \mathrm{C}_{6} \mathrm{H}_{4}\right), 8.80(\mathrm{~s}, 1 \mathrm{H}, \mathrm{NH})$. Calc. for $\mathrm{C}_{19} \mathrm{H}_{11} \mathrm{~N}_{3} \mathrm{O}_{3}(329.31): \mathrm{C}$, 69.30; H, 3.37; N, $12.76 \%$. Found: C, 69.51; H, 3.44; N, $12.81 \%$.

4-Amino-5-benzylidene-1-(1,3-dioxo-1H-inden-2(3H)-ylideneamino)-2-imino-6oxo-1,2,5,6-tetrahydropyridine-3-carbonitrile ( 8 a)

Compound $5\left(0.30 \mathrm{~g}, 9.110 \times 10^{-4} \mathrm{~mol}\right)$ was dissolved in $50 \mathrm{ml}$ of 1,4-dioxane and malononitrile $\left(0.06 \mathrm{~g}, 9.110 \times 10^{-4} \mathrm{~mol}\right)$ was added with $0.5 \mathrm{ml}$ of triethylamine as a catalyst. The reaction mixture was then heated under reflux for $3 \mathrm{hr}$ and poured on ice, a few concentrated $\mathrm{HCl}$ drops were added and stirring was carried out for a few minutes. The obtained precipitate was then collected by suction filtration.

Pale brown black crystals from ethanol, yield $69.45 \%, 0.25$ g, m.p. 288-291 ${ }^{\circ} \mathrm{C}$. IR (KBr): v/cm ${ }^{-1}=3494-3324\left(\mathrm{NH}_{2}, \mathrm{NH}\right), 3059(\mathrm{CH}$ aromatic), $2220(\mathrm{CN})$, 1692-1684 (3CO), $1669(\mathrm{C}=\mathrm{N}), 1636(\mathrm{C}=\mathrm{C}) . \mathrm{H}^{1} \mathrm{NMR}(\mathrm{DMSO}) \delta=4.88(\mathrm{~s}, 2 \mathrm{H}$, $\mathrm{NH}_{2}$ ), 6.59 (s, 1H, CH), 7.28-7.37 (m, 9H, $\left.\mathrm{C}_{6} \mathrm{H}_{5}, \mathrm{C}_{6} \mathrm{H}_{4}\right), 7.89$ (s, 1H, NH). Calc. for $\mathrm{C}_{22} \mathrm{H}_{13} \mathrm{~N}_{5} \mathrm{O}_{3}$ (395.37): C, 66.83; H, 3.31; N, 17.71\%. Found: C, 66.98; H, $3.49 ; \mathrm{N}, 17.86 \%$.

Ethyl 4-amino-5-benzylidene-1-(1,3-dioxo-1H-inden-2(3H)-ylideneamino)-2imino-6-oxo-1,2,5,6-tetrahydropyridine-3-carboxylate (8b)

Compound $5\left(0.40 \mathrm{~g}, 1.215 \times 10^{-3} \mathrm{~mol}\right)$ was dissolved in $50 \mathrm{ml}$ of 1,4-dioxane and ethyl acetoacetate $\left(0.14 \mathrm{~g}, 1.215 \times 10^{-3} \mathrm{~mol}\right)$ was added with $0.5 \mathrm{ml}$ of triethylamine as a catalyst. The reaction mixture was then heated under reflux for $2.5 \mathrm{hr}$ and poured onto ice and water, a few $\mathrm{HCl}$ (concentrated) drops were added and stirring was carried out for a few minutes. The obtained precipitate was then collected by suction filtration.

Orange crystals from ethanol yield $64.82 \%, 0.35$ g, m.p. $233-236{ }^{\circ} \mathrm{C}$. IR (KBr): $\mathrm{v} / \mathrm{cm}^{-1}=3473-3326(\mathrm{NH}), 3052\left(\mathrm{CH}\right.$ aromatic), 2987, $2879\left(\mathrm{CH}_{3}, \mathrm{CH}_{2}\right), 1679$ 1680 (4CO), $1650(\mathrm{C}=\mathrm{N}), 1637(\mathrm{C}=\mathrm{C}) . \mathrm{H}^{1} \mathrm{NMR}(\mathrm{DMSO}) \delta=1.14(\mathrm{t}, 3 \mathrm{H}, \mathrm{J}=$ $\left.7.01 \mathrm{~Hz}, \mathrm{CH}_{3}\right), 4.22\left(\mathrm{q}, 2 \mathrm{H}, \mathrm{J}=7.02 \mathrm{~Hz}, \mathrm{CH}_{2}\right), 4.80\left(\mathrm{~s}, 2 \mathrm{H}, \mathrm{NH}_{2}\right), 6.61(\mathrm{~s}, 1 \mathrm{H}$, $\mathrm{CH})$, 7.28-7.40 (m, 9H, $\left.\mathrm{C}_{6} \mathrm{H}_{5}, \mathrm{C}_{6} \mathrm{H}_{4}\right), 7.80(\mathrm{~s}, 1 \mathrm{H}, \mathrm{NH})$. Calc. for $\mathrm{C}_{24} \mathrm{H}_{18} \mathrm{~N}_{4} \mathrm{O}_{5}$ (442.42): C, 65.15; H, 4.10; N, $12.66 \%$. Found: C, 65.27; H. 4.23; N, $12.73 \%$.

5-Amino- $N^{\prime}-(1,3-$ dioxo -1 H-inden -2 (3H) -ylidene) -3-phenyl -1H -pyrazole -4carbohydrazide (10a)

To a solution of compound $5\left(0.30 \mathrm{~g}, 9.110 \times 10^{-4} \mathrm{~mol}\right)$ in $50 \mathrm{ml}$ of 1,4dioxane, hydrazine hydrate $\left(0.05 \mathrm{~g}, 9.110 \times 10^{-4} \mathrm{~mol}\right)$ was stirred in and the reaction mixture was heated under reflux for $2.5 \mathrm{hr}$, after which it was poured on ice and a few drops of concentrated $\mathrm{HCl}$ were added. The formed precipitate was collected by suction filtration.

Brown crystals from ethanol, yield $54.55 \%, 0.18$ g, m.p. $295-298^{\circ} \mathrm{C}$. IR (KBr): $\mathrm{v} / \mathrm{cm}^{-1}=3453-3320\left(\mathrm{NH}_{2}, 2 \mathrm{NH}\right), 3061$ (CH aromatic), 1690-1680 (3CO), 1663

Egypt. J. Chem. 56, No. 1 (2013) 
$(\mathrm{C}=\mathrm{N}), 1639(\mathrm{C}=\mathrm{C}) . \mathrm{H}^{1} \mathrm{NMR}(\mathrm{DMSO}) \delta=4.60\left(\mathrm{~s}, 2 \mathrm{H}, \mathrm{NH}_{2}\right), 7.31-7.48(\mathrm{~m}, 9 \mathrm{H}$, $\left.\mathrm{C}_{6} \mathrm{H}_{5}, \mathrm{C}_{6} \mathrm{H}_{4}\right), 7.77,8.81$ (2s, $\left.2 \mathrm{H}, 2 \mathrm{NH}\right)$. Calc. for $\mathrm{C}_{19} \mathrm{H}_{13} \mathrm{~N}_{5} \mathrm{O}_{3}$ (359.34): C, 63.51; H, 3.65; N, $19.49 \%$. Found: C, 63.60; H, 3.70; N, $19.68 \%$.

\section{3-Amino-N'-(1,3-dioxo-1H-inden-2(3H)-ylidene)-1,5-diphenyl-1H-pyrazole-4- carbohydrazide (10b)}

To a solution of compound $5\left(0.45 \mathrm{~g}, 1.367 \times 10^{-3} \mathrm{~mol}\right)$ in $60 \mathrm{ml}$ of $1,4-$ dioxane, phenyl hydrazine $\left(0.15 \mathrm{~g}, 1.367 \times 10^{-3} \mathrm{~mol}\right)$ was stirred in and the reaction mixture was heated under reflux for $2 \mathrm{hr}$, after which it was poured on ice and a few drops of concentrated $\mathrm{HCl}$ were added. The formed precipitate was collected by suction filtration.

Pale yellow crystals from ethanol yield $30 \%, 0.18$ g, m.p. $233-236{ }^{\circ} \mathrm{C}$. IR $(\mathrm{KBr})$ : $\mathrm{v} / \mathrm{cm}^{-1}=3483-3336(\mathrm{NH}), 3059(\mathrm{CH}$ aromatic $), 1693-1683(3 \mathrm{CO}), 1653(\mathrm{C}=\mathrm{N})$, $1640(\mathrm{C}=\mathrm{C}) . \mathrm{H}^{1} \mathrm{NMR}(\mathrm{DMSO}) \delta=4.59\left(\mathrm{~s}, 2 \mathrm{H}, \mathrm{NH}_{2}\right), 7.29-7.46\left(\mathrm{~m}, 14 \mathrm{H}, 2 \mathrm{C}_{6} \mathrm{H}_{5}\right.$, $\left.\mathrm{C}_{6} \mathrm{H}_{4}\right), 7.79$ (s, $\left.1 \mathrm{H}, \mathrm{NH}\right)$. Calc. for $\mathrm{C}_{25} \mathrm{H}_{17} \mathrm{~N}_{5} \mathrm{O}_{3}(435.43)$ : C, 68.96; H, 3.94; $\mathrm{N}$, $16.08 \%$. Found: C, 69.11; H, 4.04; N, $16.33 \%$.

2- (2- (1,3- Dioxo - 1H- inden - 2(3H) - ylidene) hydrazinyl) - 2- oxo- $N^{\prime}$ phenylacetohydrazonoyl cyanide (12)

To a solution of compound $3\left(1.11 \mathrm{~g}, 4.60 \times 10^{-3} \mathrm{~mol}\right)$ in $30 \mathrm{ml}$ of ethanol containing $0.5 \mathrm{~g}$ of sodium hydroxide pellets, benzenediazonium chloride $(0.65$ $\left.\mathrm{g}, 4.60 \times 10^{-3} \mathrm{~mol}\right)$ [prepared by adding an aqueous sodium nitrite solution $(0.32$ $\mathrm{g}, 4.60 \times 10^{-3} \mathrm{~mol}$ in $25 \mathrm{ml}$ of water $)$ to a cold solution of aniline $(0.43 \mathrm{~g}$, $4.60 \times 10^{-3} \mathrm{~mol}$ ) in $50 \mathrm{ml}$ of conc. $\mathrm{HCl}$ at $0-5^{\circ} \mathrm{C}$, with continuous stirring] was added with stirring. The reaction mixture was left for the precipitate to coagulate, and the formed precipitate was collected by suction filtration.

Light brown crystals from ethanol, yield $45.31 \%, 0.720$ g, m.p. $>300^{\circ} \mathrm{C}$. IR $(\mathrm{KBr}): \mathrm{v} / \mathrm{cm}^{-1}=3477-3326(2 \mathrm{NH}), 3063(\mathrm{CH}$ aromatic), $2256(\mathrm{CN}), 1692-1683$ (3CO), $1650(\mathrm{C}=\mathrm{N}), 1638(\mathrm{C}=\mathrm{C}) . \mathrm{H}^{1} \mathrm{NMR}(\mathrm{DMSO}) \delta=7.26-7.41\left(\mathrm{~m}, 9 \mathrm{H}, \mathrm{C}_{6} \mathrm{H}_{5}\right.$, $\left.\mathrm{C}_{6} \mathrm{H}_{4}\right), 7.77,8.78(2 \mathrm{~s}, 2 \mathrm{H}, 2 \mathrm{NH})$. Calc. for $\mathrm{C}_{18} \mathrm{H}_{11} \mathrm{~N}_{5} \mathrm{O}_{3}(345.31)$ : C, 62.61; H, 3.21 ; N, $20.28 \%$. Found: C, 62.93; H, 3.04; N, $19.98 \%$.

4-Amino-5-cyano- $N$ '-(1,3-dioxo-1H-inden-2(3H)-ylidene)-6-imino-1-phenyl-1,6dihydropyridazine-3-carbohydrazide (14a)

Compound $12\left(0.25 \mathrm{~g}, 7.24 \times 10^{-4} \mathrm{~mol}\right)$ was dissolved in $50 \mathrm{ml}$ of 1,4-dioxane containing $0.5 \mathrm{ml}$ of triethylamine. To this solution, malononitrile $(0.05 \mathrm{~g}$, $7.24 \times 10^{-4} \mathrm{~mol}$ ) has been added and the reaction mixture was then heated under reflux for $3 \mathrm{hr}$, after which it was poured on ice and a few drops of concentrated $\mathrm{HCl}$ were added. The formed precipitate was then collected by suction filtration.

Brown crystals from ethanol, yield $40 \%, 0.12$ g, m.p. $>300{ }^{\circ} \mathrm{C}$. IR $(\mathrm{KBr}): \mathrm{v} / \mathrm{cm}^{-1}=$ 3467-3328 (2NH), 3055 (CH aromatic), $2222(\mathrm{CN}), 1689-1681$ (3CO), 1651 $(\mathrm{C}=\mathrm{N}), 1643(\mathrm{C}=\mathrm{C}) . \mathrm{H}^{1} \mathrm{NMR}(\mathrm{DMSO}) \delta=4.62\left(\mathrm{~s}, 2 \mathrm{H}, \mathrm{NH}_{2}\right), 7.31-7.43(\mathrm{~m}, 9 \mathrm{H}$, 
$\left.\mathrm{C}_{6} \mathrm{H}_{5}, \mathrm{C}_{6} \mathrm{H}_{4}\right), 7.81,8.83$ (2s, 2H, 2NH). Calc. for $\mathrm{C}_{21} \mathrm{H}_{13} \mathrm{~N}_{7} \mathrm{O}_{3}$ (411.37): C, 61.31; H, 3.19; N, $23.83 \%$. Found: C, 61.57; H. 3.31; N, $23.92 \%$.

Ethyl 5-amino-6-(2-(1,3-dioxo-1H-inden-2(3H)-ylidene)hydrazinecarbonyl)-3imino-2-phenyl-2,3-dihydropyridazine-4-carboxylate(14b)

Compound $12\left(0.18 \mathrm{~g}, 5.21 \times 10^{-4} \mathrm{~mol}\right)$ was dissolved in $40 \mathrm{ml}$ of 1,4-dioxane containing $0.5 \mathrm{ml}$ triethylamine. To this solution, ethyl cyanoacetate $(0.06 \mathrm{~g}$, $\left.5.21 \times 10^{-4} \mathrm{~mol}\right)$ has been added and the reaction mixture was then heated under reflux for $2.5 \mathrm{hr}$, after which it was poured on ice and a few drops of concentrated $\mathrm{HCl}$ were added. The formed precipitate was then collected by suction filtration.

Dark brown crystals from ethanol, yield $58.33 \%, 0.14$ g, m.p. $283-286{ }^{\circ} \mathrm{C}$. IR (KBr): v/cm ${ }^{-1}=3479-3341$ (2NH), 3053 (CH aromatic), 1690-1684 (4CO), 1654 $(\mathrm{C}=\mathrm{N}), 1636(\mathrm{C}=\mathrm{C}) . \mathrm{H}^{1} \mathrm{NMR}(\mathrm{DMSO}) \delta=1.16\left(\mathrm{t}, 3 \mathrm{H}, \mathrm{J}=6.34 \mathrm{~Hz}, \mathrm{CH}_{3}\right), 4.24$ (q, 2H, J=6.34 Hz, $\left.\mathrm{CH}_{2}\right), 4.67\left(\mathrm{~s}, 2 \mathrm{H}, \mathrm{NH}_{2}\right), 7.29-7.39\left(\mathrm{~m}, 9 \mathrm{H}, \mathrm{C}_{6} \mathrm{H}_{5}, \mathrm{C}_{6} \mathrm{H}_{4}\right)$, 7.83, 8.72 (2s, $2 \mathrm{H}, 2 \mathrm{NH})$. Calc. for $\mathrm{C}_{23} \mathrm{H}_{18} \mathrm{~N}_{6} \mathrm{O}_{5}$ (458.43): C, 60.26; H, 3.96; N, $18.33 \%$. Found: C, 60.52; H, 4.02; N, $18.49 \%$.

\section{References}

1. Loncle, C., Brunel, J.M., Vidal, N., Dherbomez, M. and Letourneux, Y., Synthesis and antifungal activity of cholesterol-hydrazone derivatives. Eur. J. Med. Chem. 39, 1067 (2004).

2. Papakonstantinou-Garoufalias, S., Pouli, N., Marakos, P. and Chytyroglou-Ladas, A., Synthesis antimicrobial and antifungal activity of some new 3 substituted derivatives of 4-(2,4-dichlorophenyl)-5-adamantyl-1H-1,2,4-triazole. Farmaco, 57, 973 (2002).

3. Vicini, P., Zani, F., Cozzini, P. and Doytchinova, I., Hydrazones of 1,2benzisothiazole hydrazides: synthesis, antimicrobial activity and QSAR investigations. Eur. J. Med. Chem. 37, 553 (2002).

4. Kaushik, D., Khan, S. A., Chawla, G. and Kumar, S., N'-[(5-chloro-3-methyl-1phenyl-1 H-pyrazol-4-yl) methylene] 2/4-substituted hydrazides: Synthesis and anticonvulsant activity. Eur. J. Med. Chem. 45, 3943 (2010).

5. Sridhar, S.K., Pandeya, S.N., Stables, J.P. and Atmakuru, R., Anticonvulsant activity of hydrazones, Schiff and Mannich bases of isatin derivatives. Eur. J. Pharm. Sci. 16, 129 (2002).

6. Küçükgüzel, S.G., Mazi, A., Sahin, F., Öztürk, S. and Stables, J.P., Synthesis and biological activities of diflunisal hydrazide-hydrazones. Eur. J. Med. Chem. 38, 1005 (2003).

Egypt. J. Chem. 56, No. 1 (2013) 
7. Todeschini, A.R., Küçükgüzel, S.G., Mazi, A., Sahin, F., Öztürk, S. and Stables, J., Synthesis and biological activities of diflunisal hydrazide-hydrazones. Eur. J. Med. Chem. 38, 1005 (2003).

8. Gaston, M.A., Dias, L.R.S., Freitas, A.C.C., Miranda, A.L.P. and Barreiro, E.J., Synthesis and analgesic properties of new 4-arylhydrazone 1-H pyrazole [3,4-b] pyridine derivatives. Pharmac. Acta Helvet. 71, 213 (1996).

9. Melnyk, P., Leroux, V., Sergheraert, C. and Grellier, P., Design, synthesis and in vitro antimalarial activity of an acylhydrazone library. Bioorg. Med. Chem. Lett. 16, 31(2006).

10. Bedia, K.K., Elçin, O., Seda, U., Fatma, K., Nathaly, S., Sevim, R. and Dimoglo, A., Synthesis and characterization of novel hydrazide-hydrazones and the study of their structure-antituberculosis activity. Eur. J. Med. Chem. 41, 1253 (2006).

11. Vavř́́ková, E., Polanc, S., Kočevar, M., Horváti, K., Bősze, S., Stolaříková, J., Vávrová, K. and Vinšová, J., New fluorine-containing hydrazones active against MDR-tuberculosis. Eur. J. Med. Chem. 46, 4937 (2011).

12. Patole, J., Sandbhor, U., Padhye, S., Deobagkar, D.N., Anson, C.E. and Powell, A., Structural chemistry and In vitro antitubercular activity of acetylpyridine benzoyl hydrazone and its copper complex against Mycobacterium smegmatis. Bioorg. Med. Chem. Lett. 13, 51 (2003).

13. Maccari, R., Ottanà, R. and Vigorita, M.G., Part 14, In vitro advanced antimycobacterial screening of isoniazid-related hydrazones, hydrazides and cyanoboranes. Bioorg. Med. Chem. Lett. 15, 2509 (2005).

14. Pavan, F.R., Maia, P.I.S., Leite, S.R.A., Deflon, V.M., Batista, A.A., Sato, D.N., Franzblau, S.G. and Leite, C.Q.F., Thiosemicarbazones, semicarbazones, dithiocarbazates and hydrazide/hydrazones: Anti - Mycobacterium tuberculosis activity and cytotoxicity. Eur. J. Med. Chem. 45, 1898-1905 (2010).

15. Karalı, N., Kocabalkanlı, A., Gürsoy, A. and Ateş, Ö., Synthesis and antitubercular activity of 4-(3-coumarinyl)-3-cyclohexyl-4-thiazolin-2-one benzylidenehydrazones. Farmaco, 57, 589-93 (2002).

16. Mohareb, R. and Al-Omran, F., Reaction of pregnenolone with cyanoacetylhydrazine: Novel synthesis of hydrazine-hydrazone, pyrazole, pyridine, thiazole, thiophene derivatives and their cytotoxicity evaluations. Steroids, 17, 1551(2012).

17. Cosgrove, K.L. and McGeary, R.P., Exploring the versatility of the Johnson-Claisen rearrangement: Access to functionally versatile $\delta$-ethoxycarbonyl- $\alpha, \beta$-unsaturated nitriles. Tetrahedron, 66, 3050 (2010). 
18. Elison, M.N., Feducovich, S.K., Starikova, ZA., Vereshchagin, A.N. and Nikishin, G.I., Stereoselective electrocatalytic transformation of arylidenemalonotriles and malononitrile into (1R,5S,6R)-6-aryl-2-amino-4,4-dialkoxy-1,-5-dicyano-3-azabicyclo [3.1.0] hex-2-ens. Tetrahedron, 60, 11743 (2004).

19. Behbehani, H., Ibrahim, H.M., Makhseed, S., Elnagdi, M.H. and Mahmoud, H., 2-Aminothiophenes as building blocks in heterocyclic synthesis: Synthesis and antimicrobial evaluation of a new class of pyrido [1,2-a] thieno [3,2-c]pyrimidine, quinoline and pyridine-2-one derivatives. Eur. J. Med. Chem. 52, 51 (2012).

(Received 7/3/2013;

accepted 15/4/2013)

$$
\begin{aligned}
& \text { السمية لمشتقات الهيدرايزيد - هيدرازون تجاه خطوط الخلايا } \\
& \text { السرطانية و الطبيعية } \\
& \text { ميسون يوسف زكى } \\
& \text { الهيئة القومية للرقابة و البحوث الدو ائية ـ القاهرة - مصر. }
\end{aligned}
$$

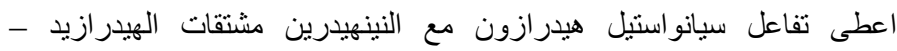

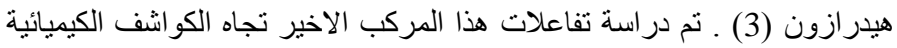

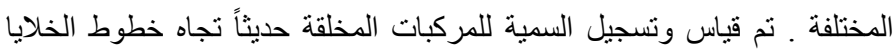

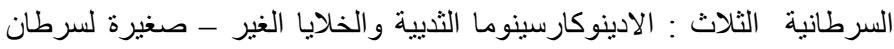

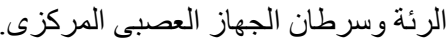

